\title{
Staphylococcal aureus Enterotoxin C and Enterotoxin-Like L Associated with Post-partum Mastitis
}

\author{
Kristina T. Franck ${ }^{1 *}$, Heidi Gumpert ${ }^{2}$, Bente Olesen ${ }^{1}$, Anders R. Larsen ${ }^{3}$, \\ Andreas Petersen ${ }^{3}$, Jette Bangsborg ${ }^{1}$, Per Albertsen ${ }^{4}$, Henrik Westh ${ }^{2,5}$ and \\ Mette D. Bartels ${ }^{2}$ \\ ${ }^{1}$ Department of Clinical Microbiology, Herlev and Gentofte Hospital, University of Copenhagen, Herlev, Denmark, \\ ${ }^{2}$ Department of Clinical Microbiology, Hvidovre Hospital, University of Copenhagen, Hvidovre, Denmark, ${ }^{3}$ Reference \\ Laboratory for Antimicrobial Resistance and Staphylococci, Statens Serum Institut, Copenhagen, Denmark, ${ }^{4}$ Department of \\ Pediatrics, Nordsjællands Hospital, University of Copenhagen, Hillerød, Denmark, ${ }^{5}$ Faculty of Health Sciences, Institute of \\ Clinical Medicine, University of Copenhagen, Copenhagen, Denmark
}

Denmark is a low prevalence country with regard to methicillin resistant Staphylococcus aureus (MRSA). In 2008 and 2014, two neonatal wards in the Copenhagen area experienced outbreaks with a typical community acquired MRSA belonging to the same spa type and sequence type (t015:ST45) and both were PVL and ACME negative. In outbreak 1, the isolates harbored SCCmec IVa and in outbreak 2 SCCmec V. The clinical presentation differed between the two outbreaks, as none of five MRSA positive mothers in outbreak 1 had mastitis vs. five of six MRSA positive mothers in outbreak $2(p<0.02)$. To investigate if whole-genome sequencing could identify virulence genes associated with mastitis, t015:ST45 isolates from Denmark ( $N=101)$ were whole-genome sequenced. Sequence analysis confirmed two separate outbreaks with no sign of sustained spread into the community. Analysis of the accessory genome between isolates from the two outbreaks revealed a $S$. aureus pathogenicity island containing enterotoxin $C$ and enterotoxin-like $L$ only in isolates from outbreak 2. Enterotoxin $C$ and enterotoxin-like $L$ carrying $S$. aureus are associated with bovine mastitis and our findings indicate that these may also be important virulence factors for human mastitis.

Keywords: SaPITokyo12571, enterotoxins, clinical presentation, post-partum mastitis, pathogenicity islands, MRSA, whole-genome sequencing, virulence

\section{INTRODUCTION}

Methicillin resistant Staphylococcus aureus (MRSA) is endemic in hospitals worldwide and an increasing challenge in the community. In Denmark, despite a rise in MRSA in the last decade, the prevalence of MRSA is low and is dominated by community-acquired and livestock-associated MRSA (Bager et al., 2015). When MRSA outbreaks occur in Danish hospitals, they are primarily in neonatal wards, where asymptomatic carriage as well as infection is encountered in both neonates and their mothers. The index case, in most outbreaks, is typically a neonate with a minor infection such as conjunctivitis, with the MRSA most likely acquired from a parent. In the Capital Region of Denmark, all MRSA isolates are whole-genome sequenced and spa-types, multilocus sequence types (MLST) plus the presence or absence of the Panton-Valentine leukocidin (PVL) genes are reported to the clinicians (Bartels et al., 2015). Apart from the PVL genes, isolates are not routinely examined for other virulence genes such as enterotoxins and exfoliative toxins (Peacock et al., 2002). 
We analyzed the genomes of two seemingly similar neonatal MRSA outbreaks caused by MRSA t015:ST45. The clinical presentation of the two outbreaks was, however, very different. Mothers in the first outbreak (O1) were only colonized with MRSA whereas most mothers in the second outbreak (O2) suffered from mastitis caused by MRSA. Outbreaks of postpartum mastitis or post-partum breast abscesses are rarely reported (Saiman et al., 2003; Manoharan et al., 2012) although cohort studies show that $8-20 \%$ of breastfeeding women may suffer from mastitis (Scott et al., 2008; Cullinane et al., 2015; Khanal et al., 2015).

\section{MATERIALS AND METHODS}

\section{Setting}

Both outbreaks occurred in the greater Copenhagen area, the Capital Region of Denmark, in 2008 and 2014, respectively, in two different hospital neonatal wards. Isolates were found in clinical samples (one case of conjunctivitis, five breast milk samples from patients with mastitis) or through screening for colonization. O1 involved 9 neonates, 5 mothers, and 4 fathers while $\mathrm{O} 2$ involved 12 neonates, 6 mothers, and 2 hospital staff members. Fathers were not tested for MRSA in O2. In both outbreaks, MRSA positive neonates and parents were contact isolated. Both wards were disinfected with manual bleach cleaning followed by a hydrogen-peroxide disinfectant mist. MRSA colonized staff members went on medical leave and underwent successful decolonization therapy. The neonates and their parents were not offered decolonization therapy in accordance with the Danish National Guidelines, recommending no treatment until the child turns 2 years (Vejledning om forebyggelse af spredning af MRSA, 2016).

\section{Data Set}

MRSA isolates from the outbreaks were investigated by wholegenome sequencing (WGS) as part of the routine at the Department of Clinical Microbiology, Hvidovre Hospital. In order to investigate the relatedness to other Danish MRSA with spa-type t015 isolated in 2008-15, WGS from isolates obtained through the national surveillance at Statens Serum Institut, were included in the analysis. The combined data set included 102 patient unique t015:ST45 isolates from all over Denmark isolated in the period 2008-2015. One isolate was excluded due to poor sequencing quality.

\section{Whole-Genome Sequencing and Analysis}

DNA was extracted from isolates and sequencing libraries were prepared using Illumina Nextera XT protocol, according to the manufacturer (Illumina, CA, USA). Isolates were assembled using Velvet (Zerbino and Birney, 2008) with VelvetOptimizer. Isolates were annotated using PROKKA (Seemann, 2014), and the core- and pan-genomes were determined using default values. The aligned, ungapped core genome was used for SNP calling and phylogenetic analysis was performed by RAxML with 100 bootstrap support replicates (Stamatakis, 2014). The resulting phylogenetic tree and accessory genome (non-unique genes present in $<70 \%$ of isolates) was visualized in $\mathrm{R}$ using ggplots2 and ggtree packages (Yu et al., 2017). The columns in the heatmap were ordered by a hierarchical clustering algorithm based on gene presence/absence in the accessory genome using the Jaccard distance metric.

In addition to the pan-genome analysis, regions unique to either of the outbreaks were identified as follows. Reads from the index isolate from $\mathrm{O} 1$ were aligned to the genome assembly of the index isolate from $\mathrm{O} 2$ using Bowtie2 (Langmead and Salzberg, 2012), and vice versa. Coverage breadth of the read alignment was established using BedTools (Quinlan and Hall, 2010) for coding regions identified from a RAST annotation (Aziz et al., 2008). The coverage breadth output was filtered such that at least $90 \%$ coverage breadth of the coding region was required for the gene to be considered present. In this way, consecutive regions of coverage breadth $<90 \%$ were identified and represented unique genomic regions to one of the index outbreak isolates. The same analysis was performed for the isolates within each outbreak to the index isolate of the outbreak to identify any other difference over the course of the respective outbreaks.

The spa, MLST, PVL genes, and SCCmec type were extracted from WGS data, as previously described (Bartels et al., 2015). Virulence and resistance genes and plasmid replicons were identified with the online tools VirulenceFinder (Joensen et al., 2014), ResFinder (Zankari et al., 2012), and PlasmidFinder (Carattoli et al., 2014), respectively, available from the homepage http://genomicepidemiology.org/.

The sequences of the index isolates from each outbreak have been deposited at NCBI under WGS accession numbers MPPG00000000 and MPPH00000000.

\section{Statistical Analysis}

Fisher's exact test was used for comparison of the proportions of mastitis among mothers in the two outbreaks.

\section{Ethical Considerations}

The study was approved by the Danish Data Protection Agency (no. 2012-58-0004).

\section{RESULTS}

\section{MRSA Colonization and Infection}

In $\mathrm{O} 1$, the index case was a neonate who tested MRSA positive in a conjunctivitis sample. The remaining 17 MRSA positive cases were colonized without infection and there were no reports of mastitis in the five MRSA positive mothers. In O2, the index case was a mother with mastitis. MRSA as well as MSSA was detected in a sample of her breast milk. Twelve neonates, six mothers and two staff members tested MRSA positive. A significant larger proportion of the MRSA positive mothers in O2 suffered from early onset mastitis based on results from culturing of breast milk samples $(5 / 6)$ compared to MRSA positive mothers in $\mathrm{O} 1(0 / 5)$ $(p<0.02)$.

\section{Whole-Genome Sequencing Results}

High quality WGS data were obtained for 101 (99\%) of the MRSA isolates. All isolates were confirmed to be spa type t015:ST45. No isolates were PVL-positive. A phylogenetic tree based on 


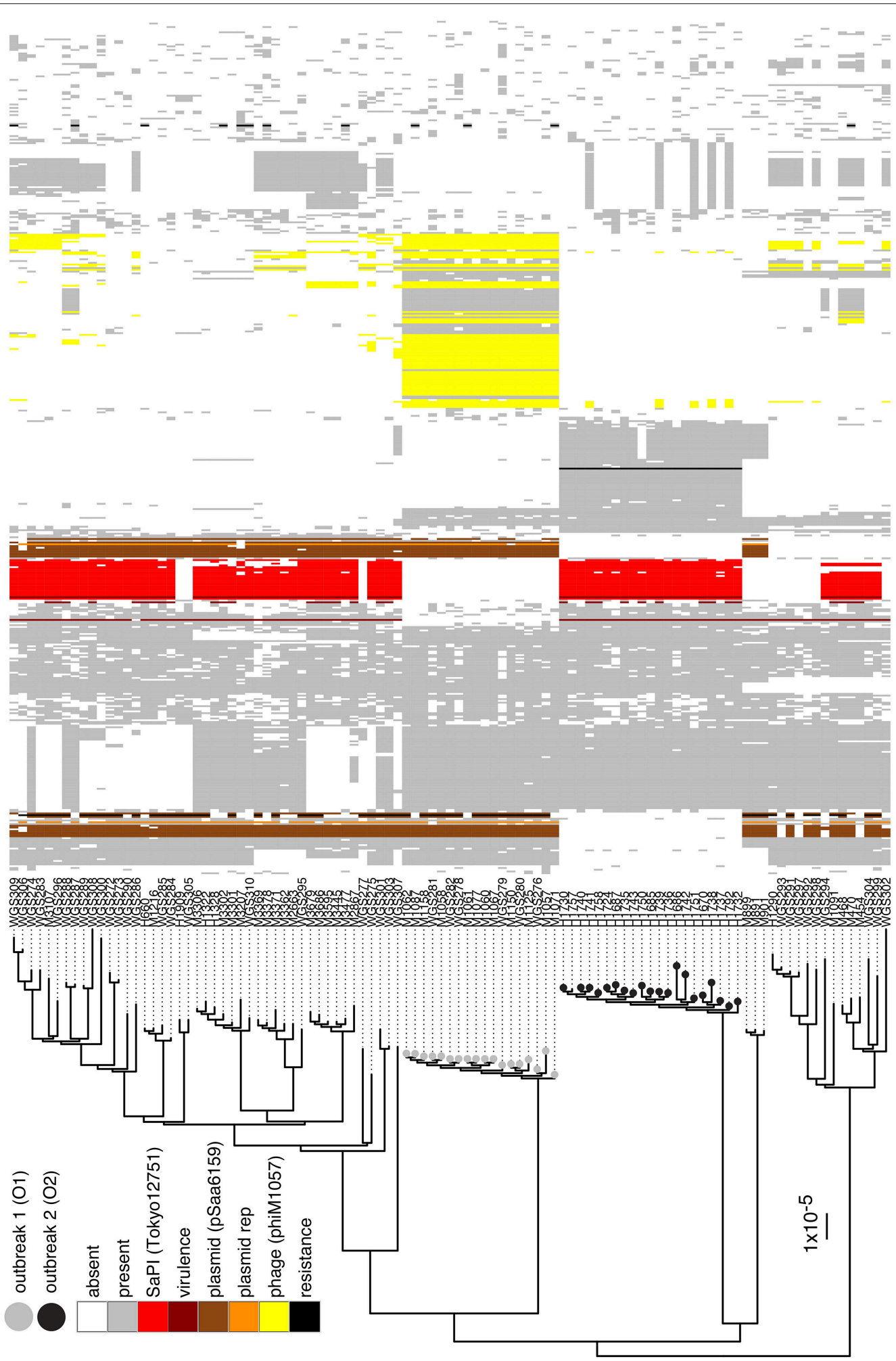

FIGURE 1 | Phylogenetic tree and accessory genome heatmap. On the left hand side is a maximum-likelihood-based phylogenetic tree based on the variable positions in the core-genome of the t015:ST45 isolates from Denmark during 2008-2015. Scale bar indicates the substitution rate. Isolates with a gray circle belong to $\mathrm{O} 1$ and black circle to $\mathrm{O} 2$. On the right is a heat map of the accessory genome of $\mathrm{O} 1$ and $\mathrm{O} 2$ isolates, where the accessory genome is defined as the genes that are present in at most $70 \%$ of the isolates. Columns are sorted by a hierarchal clustering algorithm based on gene absence/presence in the $\mathrm{O} 1$ and $\mathrm{O} 2$ accessory genome. Genes that have been identified to belong to the SaPI present in O2, the multi-replicon plasmid in $\mathrm{O} 1$, phages in $\mathrm{O} 1$ and $\mathrm{O} 2$ are colored, along with virulence and resistance genes and plasmid replicons identified by VirulenceFinder, ResFinder, and PlasmidFinder, respectively. 
the ungapped alignment of core-genome (2095 genes totaling $1,655,427 \mathrm{bp}$ ) of the isolates was produced to investigate the relationship between the isolates (Figure 1). The average number of base pair substitutions in the core genome of all the isolates was 340. Isolates from $\mathrm{O} 1$ and $\mathrm{O} 2$ grouped into two distinct, separate clusters with isolates within $\mathrm{O} 1$ and $\mathrm{O} 2$ differing by no more than 22 and 44 positions, respectively. There were a minimum of 420 variable positions in the core genome between isolates from $\mathrm{O} 1$ and $\mathrm{O} 2$. The $\mathrm{O} 1$ cluster contained only isolates from known outbreak patients, whereas an additional isolate sampled from a woman with no known contact to the outbreak grouped into the $\mathrm{O} 2$ cluster (H1757). This patient was a 53 year old woman who lived in the catchment area of the hospital, where O2 occurred.

We further investigated whether differences in the genomic content between isolates of the two outbreaks might contribute to the enhanced virulence (based on the number of mastitis cases) observed in $\mathrm{O} 2$ compared to $\mathrm{O} 1$. Isolates from $\mathrm{O} 2$ were found to harbor SaPITokyo12571 (Suzuki et al., 2015), a pathogenicity island that contained genes encoding staphylococcal enterotoxin C (SEC) and staphylococcal enterotoxin-like L (SElL; Figure 1). VirulenceFinder identified the sec and sell genes as the only difference in known virulence factors between the two outbreak index isolates (Supplementary Table). Further analysis of 4695 $S$. aureus genomes in our database revealed that 562 isolates (12\%) harbored both sec and sell genes while only 10 isolates (0.2\%) had sell alone. Among the 562 isolates, 120 belonged to ST45 and $71 \%$ of all ST45 isolates in the complete WGS database harbored sec and sell. None of the ST45 isolates harbored the PVL genes. Isolates from O1 harbored a rep5rep16 multi-replicon $20.8 \mathrm{kbp}$ plasmid that shared 99\% identity with pSaa6159 (Chua et al., 2010). This plasmid harbored cadmium resistance genes, and blaZ beta-lactamase gene with corresponding blaR1 and blaI regulatory genes. Additionally, O1 isolates harbored an extra $42.8 \mathrm{kbp}$ on the chromosome relative to $\mathrm{O} 2$ isolates due to insertion of a $33.8 \mathrm{kbp}$ prophage and partly from the different sizes of the two SCCmec cassettes (SCCmec IVa in $\mathrm{O} 1$ and SCCmec $\mathrm{V}$ in O2). This prophage, phiM1057, most closely resembles bacteriophage 29, (GenBank: AY954964) with a coverage of about $80 \%$ and also has the same integrase.

The distribution of the accessory genome of the $\mathrm{O} 1$ and $\mathrm{O} 2$ isolates across all t015 isolates in the study revealed that while some elements were unique to one of the outbreaks (e.g., phiM1057 in O1), others were relatively widespread across the other t015 isolates (e.g., the multireplicon plasmid from $\mathrm{O} 1$ and the SaPITokyo12571 from O2; Figure 1).

\section{DISCUSSION}

Human outbreaks of post-partum mastitis and transmission between mothers with MRSA in breast milk and neonates have been described previously (Kawada et al., 2003; Saiman et al., 2003; Behari et al., 2004; Sanchini et al., 2013). In these outbreaks, the infecting $S$. aureus strains expressed the PVL genes, a known cause of skin and soft tissue infections. None of our outbreak strains contained the PVL genes. The two outbreaks, although having identical spa-type and ST (t015:ST45), were found to be caused by different MRSA and a possible cause of mastitis in O2 was identified as a SaPI similar to SaPITokyo12571. This SaPI contained sec and sell, which have both been described as individual causes of mastitis in cows (Kenny et al., 1993; Orwin et al., 2003; Artursson et al., 2016). SEC has also been detected in a PVL containing MRSA strain causing an outbreak of skin-and soft tissue infections including mastitis among post-partum women (Saiman et al., 2003). sec and sell were the only additional known virulence genes identified by VirulenceFinder in $\mathrm{O} 2$ compared to O1. Our finding that sec and sell are in the genome of $12 \%$ of our MRSA isolates, with sell alone in $0.2 \%$, suggests a connection between either or both enterotoxins with the development of mastitis in women. Interestingly, sec and sell were found in $71 \%$ of the ST45 isolates in our WGS database and none of the ST45 isolates harbored the PVL genes. sec and sell were found to be seven times more common in ST45 than in the remaining MRSA clonal complexes. The prophage, phiM1057, identified in O1, was in contrast only associated to this outbreak and not seen in other ST45 isolates. The plasmid, pSaa6159, was common in ST45 isolates but not found in $\mathrm{O} 2$ and contained no known virulence genes.

With this study, we used WGS on nationally collected MRSA strains to document the containment of two outbreaks of MRSA in two neonatal wards. In one outbreak, the majority of mothers developed mastitis, which could be associated with the presence of a $S$. aureus pathogenicity island containing sec and sell in the outbreak strain. Further analysis of WGS data in future outbreaks can pinpoint genes that can cause specific clinical presentations.

\section{AUTHOR CONTRIBUTIONS}

KF, BO, AL, AP, HW, MB: conceived the study. KF, HG, AL, AP, $\mathrm{HW}, \mathrm{MB}$ : analyzed and interpreted the results. KF, BO, AL, AP, JB, PA, HW, MB: collected and interpreted data. KF, HW: drafted the manuscript, which was critically revised and final approved by all the authors.

\section{ACKNOWLEDGMENTS}

We thank the Departments of Clinical Microbiology in Denmark, who have contributed with newly diagnosed MRSA strains to the national surveillance program at Statens Serum Institut. Henrike Zschach identified the phage and Lone Dons the SaPI.

\section{SUPPLEMENTARY MATERIAL}

The Supplementary Material for this article can be found online at: http://journal.frontiersin.org/article/10.3389/fmicb. 2017.00173/full\#supplementary-material 


\section{REFERENCES}

Artursson, K., Söderlund, R., Liu, L., Monecke, S., and Schelin, J. (2016). Genotyping of Staphylococcus aureus in bovine mastitis and correlation to phenotypic characteristics. Vet. Microbiol. 193, 156-161. doi: 10.1016/j.vetmic.2016.08.012

Aziz, R. K., Bartels, D., Best, A. A., DeJongh, M., Disz, T., Edwards, R. A., et al. (2008). The RAST Server: rapid annotations using subsystems technology. BMC Genomics 9:75. doi: 10.1186/14712164-9-75

Bager, F., Birk, T., Høg, B. B., Jensen, L. B., Jensen, A. N., de Knegt, L., et al. (2015). DANMAP 2014 - Use of Antimicrobial Agents and Occurence of Antimicrobial Resistence in Bacteria from Food Animals, Food and Humans in Denmark. Copenhagen: GraphicCo.

Bartels, M. D., Larner-Svensson, H., Meiniche, H., Kristoffersen, K., Schønning, K., Nielsen, J. B., et al. (2015). Monitoring meticillin resistant Staphylococcus aureus and its spread in Copenhagen, Denmark, 2013, through routine whole genome sequencing. Euro Surveill. 20, 20-28. doi: 10.2807/1560-7917.ES2015.20.17.21112

Behari, P., Englund, J., Alcasid, G., Garcia-Houchins, S., and Weber, S. G. (2004). Transmission of methicillin-resistant Staphylococcus aureus to preterm infants through breast milk. Infect. Control Hosp. Epidemiol. 25, 778-780. doi: $10.1086 / 502476$

Carattoli, A., Zankari, E., García-Fernández, A., Larsen, M. V., Lund, O., Villa, L., et al. (2014). In silico detection and typing of plasmids using PlasmidFinder and plasmid multilocus sequence typing. Antimicrob. Agents Chemother. 58, 3895-3903. doi: 10.1128/AAC.02412-14

Chua, K., Seemann, T., Harrison, P. F., Davies, J. K., Coutts, S. J., Chen, H., et al. (2010). Complete genome sequence of Staphylococcus aureus strain JKD6159, a unique Australian clone of ST93-IV community methicillinresistant Staphylococcus aureus. J. Bacteriol. 192, 5556-5557. doi: 10.1128/JB.00 $878-10$

Cullinane, M., Amir, L. H., Donath, S. M., Garland, S. M., Tabrizi, S. N., Payne, M. S., et al. (2015). Determinants of mastitis in women in the CASTLE study: a cohort study. BMC Fam. Pract. 16:181. doi: 10.1186/s12875-015-0396-5

Joensen, K. G., Scheutz, F., Lund, O., Hasman, H., Kaas, R. S., Nielsen, E. M., et al. (2014). Real-time whole-genome sequencing for routine typing, surveillance, and outbreak detection of verotoxigenic Escherichia coli. J. Clin. Microbiol. 52, 1501-1510. doi: 10.1128/JCM.03617-13

Kawada, M., Okuzumi, K., Hitomi, S., and Sugishita, C. (2003). Transmission of Staphylococcus aureus between healthy, lactating mothers and their infants by breastfeeding. J. Hum. Lact. 19, 411-417. doi: 10.1177/08903344032 57799

Kenny, K., Reiser, R. F., Bastida-Corcuera, F. D., and Norcross, N. L. (1993). Production of enterotoxins and toxic shock syndrome toxin by bovine mammary isolates of Staphylococcus aureus. J. Clin. Microbiol. 31, 706-707.

Khanal, V., Scott, J. A., Lee, A. H., and Binns, C. W. (2015). Incidence of mastitis in the neonatal period in a traditional breastfeeding society: results of a cohort study. Breastfeed. Med. 10, 481-487. doi: 10.1089/bfm.2015.0080

Langmead, B., and Salzberg, S. L. (2012). Fast gapped-read alignment with Bowtie 2. Nat. Methods 9, 357-359. doi: 10.1038/nmeth.1923

Manoharan, A., Zhang, L., Poojary, A., Bhandarkar, L., Koppikar, G., and Robinson, D. A. (2012). An outbreak of post-partum breast abscesses in Mumbai, India caused by ST22-MRSA-IV: genetic characteristics and epidemiological implications. Epidemiol. Infect. 140, 1809-1812. doi: $10.1017 /$ S0950268812000593
Orwin, P. M., Fitzgerald, J. R., Leung, D. Y., Gutierrez, J. A., Bohach, G. A., and Schlievert, P. M. (2003). Characterization of Staphylococcus aureus enterotoxin L. Infect. Immun. 71, 2916-2919. doi: 10.1128/IAI.71.5.2916-2919.2003

Peacock, S. J., Moore, C. E., Justice, A., Kantzanou, M., Story, L., Mackie, K., et al. (2002). Virulent combinations of adhesin and toxin genes in natural populations of Staphylococcus aureus. Infect. Immun. 70, 4987-4996. doi: 10.1128/IAI.70.9.4987-4996.2002

Quinlan, A. R., and Hall, I. M. (2010). BEDTools: a flexible suite of utilities for comparing genomic features. Bioinformatics 26, 841-842. doi: 10.1093/bioinformatics/btq033

Saiman, L., O'Keefe, M., Graham, P. L., Wu, F., Saïd-Salim, B., Kreiswirth, B., et al. (2003). Hospital transmission of community-acquired methicillinresistant Staphylococcus aureus among postpartum women. Clin. Infect. Dis. 37, 1313-1319. doi: 10.1086/379022

Sanchini, A., Spitoni, M. G., Monaco, M., Raglio, A., Grigis, A., Petrò W., et al. (2013). Outbreak of skin and soft tissue infections in a hospital newborn nursery in Italy due to community-acquired meticillin-resistant Staphylococcus aureus USA300 clone. J. Hosp. Infect. 83, 36-40. doi: 10.1016/j.jhin.2012. 09.017

Scott, J. A., Robertson, M., Fitzpatrick, J., Knight, C., and Mulholland, S. (2008). Occurrence of lactational mastitis and medical management: a prospective cohort study in Glasgow. Int. Breastfeed. J. 3:21. doi: 10.1186/1746-4358-3-21

Seemann, T. (2014). Prokka: rapid prokaryotic genome annotation. Bioinformatics 30, 2068-2069. doi: 10.1093/bioinformatics/btu153

Stamatakis, A. (2014). RAxML version 8: a tool for phylogenetic analysis and post-analysis of large phylogenies. Bioinformatics 30, 1312-1313. doi: 10.1093/bioinformatics/btu033

Suzuki, Y., Kubota, H., Sato'o, Y., Ono, H. K., Kato, R., Sadamasu, K., et al. (2015). Identification and characterization of novel Staphylococcus aureus pathogenicity islands encoding staphylococcal enterotoxins originating from staphylococcal food poisoning isolates. J. Appl. Microbiol. 118, 1507-1520. doi: $10.1111 /$ jam. 12786

Vejledning om forebyggelse af spredning af, MRSA. (2016). Danish Health Authority.

Yu, G., Smith, D. K., Zhu, H., Guan, Y., and Lam, T. T.-Y. (2017). GGTREE: an $r$ package for visualization and annotation of phylogenetic trees with their covariates and other associated data. Methods Ecol. Evol. 8, 28-36. doi: 10.1111/2041-210X.12628

Zankari, E., Hasman, H., Cosentino, S., Vestergaard, M., Rasmussen, S., Lund, O., et al. (2012). Identification of acquired antimicrobial resistance genes. J. Antimicrob. Chemother. 67, 2640-2644. doi: 10.1093/jac/dks261

Zerbino, D. R., and Birney, E. (2008). Velvet: algorithms for de novo short read assembly using de Bruijn graphs. Genome Res. 18, 821-829. doi: $10.1101 /$ gr.074492.107

Conflict of Interest Statement: The authors declare that the research was conducted in the absence of any commercial or financial relationships that could be construed as a potential conflict of interest.

Copyright (c) 2017 Franck, Gumpert, Olesen, Larsen, Petersen, Bangsborg, Albertsen, Westh and Bartels. This is an open-access article distributed under the terms of the Creative Commons Attribution License (CC BY). The use, distribution or reproduction in other forums is permitted, provided the original author(s) or licensor are credited and that the original publication in this journal is cited, in accordance with accepted academic practice. No use, distribution or reproduction is permitted which does not comply with these terms. 